\title{
Sammelbericht aus dem Karst ${ }^{1}$ ). \\ Von
}

Schulrath Dr. Eg. Schreiber in Görz.

Orinocarabus carinthiacus St. Im Tarnowanergebirge von 1000 Met. aufwärts, besonders an Waldrändern und in aufgelassenen Waldwegen, nicht selten.

Carabus montivagus Pall. In Gesellschaft des vorigen, aber etwas häufiger.

Car. italicus Bon. Um St. Peter bei Görz gesammelt, selten.

Nebria psammodes Rossi. An Bachufern um Görz, mitunter häufig. Trechus laevipennis Heer. In den jul. Alpen, von 2000 M. an, s. h. Adialampus arenarius Bon. Einige Ex. am Meeresufer bei Monfalcone unter Genist.

Chlaenius velutinus Dft. Um Görz u. an den südl. Karstseen, gemein. Chl. variegalus Fourc. Um Görz in den Sümpfen von Podgora und am Doberdoer See stellenweise sehr häufig.

Chl. azureus Dft. Um Görz und im südl. Karst auf trockenen Wiesen, nicht häufig.

Acinopus picipes $\mathrm{Ol}$. Im südl. Karst, stellenweise nicht selten.

Myas chalybaeus Pall. Im südl. Karst, besonders um Nabresina in den sog. Dolinen (kesselförmigen Vertiefungen), eben nicht selten. - Uebrigens auch in Istrien, z. B. um Pola.

Lianoë planipennis Schchl. In den jul. Alpen von 1500 Metern an weit verbreitet, aber nicht häufig.

Molops striolatus $\mathrm{Fb}$. und ovipennis Chaud. In Gebirgswäldern bis gegen 1200 Meter hinauf, allenthalben gemein.

Pterostichus Welensii Drap. Im Tarnowanerwalde, aber nicht bäufig. Pt. Ziegleri Dft. In den jul. Alpen von 1000-2000 M., sehr häufig. Pt. hungaricus Dej. Um Görz, gemein.

Pt. elongatus Dft. Am Doberdoer See im südl. Karst, selten.

Pt. cognatus Dej. In den jul. Alpen, sehr häufig.

Stomis rostratus St. Im Tarnowaner Gebirge, sebr vereinzelt.

1) Ich lasse diesen Sammelbericht fast unverändert folgen, da er den norddeutschen Coleopterologen von besonderem Interesse sein dürfte.

Dr. G. Kraatz. 
Antisphodrus Schreibersii Küst. In Karstlöchern überall, aber nicht h. Ant. elegans Dej. Um Görz u. im Tarnowanerwalde unter Steinen, s. s. Laemostenus janthinus Dst. Im Tarnowaner Gebirge, nicht selten. Laem. elongatus Dej. Im nördl. Karst, nicht selten, in Kellern oft in Menge.

Calathus glabricollis Dej. Im ganzen Karst, nicht selten.

Lebia pubipennis Duf. Im Karst einzeln, kaum über 300 Meter hoch hinauf.

Cymindis coadunata Dej. Im Karst die gewöhnlichste Art.

Odocantha melanura L. In den Sümpfen längs der Bahn um Görz, nicht selten.

Hydrovatus cuspidatus Kunz, ebenda, selten.

Bidessus 2-carinatus Str. Im Algengewirr in Wassergräben um Görz, sehr häufig.

Deronectes luctuosus Aub. Im Isonzo um Görz, stellenweise z. h. Hydroporus Dawisii Curt. Ebenda vom Ursprung bis Görz, s. h. Hydr. vagepictus Fairm. In -den Bahnsümpfen, besonders nach Süden zu, häufig.

Agabus Solieri Aub. In Hochgebirgsseen massenhaft.

Gyrinus 2-color Payk. Einzeln um Görz.

Gyr. caspius Mentr. Im October stellenweise die einzige Art u. s. h. Dryops algirica Luc. Am Isonzo um Görz, sehr gemein.

Potaminus substriatus Müll. An Ufern kalter Bäche um Görz, stellenweise häufig.

Heterocerus murinus Kiesw. Am Isonzo bei Tolmein, sehr häufig. Velleius dilatatus Fab. 2 Ex. am Eingange eines Horniss-Nestes an einer Eiche bei Görz.

Astrapaeus ulmi Rossi. Im Spätherbst in den Feldern gegen den Isonzo, nicht sehr selten.

Staphylinus lutarius Grv. Im südl. Karst in Kuhmist, häufig.

Sunius uniformis Duv. Um Görz, selten.

Paederus sanguinicollis Steph. Am Isonzo bei Görz, äufserst gemein. Thinodromus dilatatus Er. Ebenda, häufig.

Centrotoma lucifuga Heyd. Um Görz, aber selten.

Ctenistes palpalis Rchb. Um Görz, gemein.

Necrophorus nigricornis Falderm. Im Tarnowaner Gebirge von 1000 Metern an auf waldfreien Stellen, sehr häufig.

Psammoechus 2-punctatus Fb. In den Bahnsümpfen um Görz, n. s. Anthrenus molitor Aub. Um Cormons auf Blüthen, selten.

Saprinus maculatus Rss. 1 Ex. um Cormons im Fluge.

Ateuchus sacer L. 1 Ex. bei Monfalcone, 2 Ex. um Görz. 
Anthophagus amyntas $\mathrm{Ol}$. Im südl. Karst in Kuhdünger, sehr häufig. Triodonta nitidula Rss. Im Juni auf sandigen Wiesen am Isonzo bei Görz, sehr häufig.

Rhizotrogus fraxinicola $\mathrm{Hgb}$. Im südl. Karst, im April spät Abends häufig schwärmend.

Anoxia matutinalis Sap. Im nördl. Karst mitunter in Menge auftretend.

Acmaeodera crinita Gory. Im südl. Karst unter Rinde von Quercus ilex, nicht häufig.

Aphanisticus elongatus Vill. In den Lagunen bei Monfalcone auf Binsen besonders im Mai, sehr häufig.

Elater erythrogonus Müll. Im Tarnowanerwalde unter Rinden verfaulender Bäume.

Cryptohypnus frigidus Kiesw. In den jul. Alpen in morschen Bäumen, nicht selten.

Melanotus tenebrosus Er. Im Karst, gemein.

Ludius occitanicus Vill. Auf Weiden im südl. Karst. Riesige Stücke. Malchinus demissus Kiesw. Auf Hecken unmittelbar hinter der Stadt, häufig.

Colotes maculatus Lap. In den Lagunen der Adria, ziemlich bäufig, besonders auf Binsen.

Dasytes tardus Schauf. Auf Blüthen um Görz, mitunter häufig. Haplocnemus pulverulentus Küst. Im nördl. Karst auf Blüthen von Scrophularia canina und Athamantha Matthioli, am häufigsten um 1000 Meter Höhe herum.

Tarsostenus 1-vittatus Rss. Bei Holzlagern in der Stadt Abends fliegend.

Mezium sulcatum $\mathrm{Fb}$. und affine Bld. An Häusern, aber selten. Sinoxylon 6-dentatum Ol. In den $\mathrm{Z}$ weigen von Paliurus aculeatus stellenweise häufig.

Stenosis brenthoides Rss. Unter Genist und Graswurzeln am Meeresufer, nicht selten.

Blaps gages L. Um Görz selten, im südl. Karst aber häufig. Asida grisea $\mathrm{Fb}$. Im ganzen Karst bis $1000 \mathrm{M}$. boch, nicht selten. Phaleria hemisphaerica Küst. Am Meeresufer nicht selten.

Laena pimelia $\mathrm{Fb}$. Um Görz unter Laub an Hecken, selten. Acanthopus caraboides Ptg. Im Karst unter Moos an Baumstrünken, selten.

Helops coeruleus L. Um Görz allenthalben, besonders in alten Weiden; aber nicht häufig.

Hel. Rossii Grm. Im südl. Karst, unter Baumrinden oft nesterweise. 
Stenomax exaratus Grm. Um Görz und selbst in der Stadt allenthalben, nicht selten.

Upinella aterrima Küst. Besonders an ausgegrabenen Wurzeln der Maulbeerbäume in den ersten Morgenstunden herumlaufend; bei Tage selten zu sehen.

Omophlus rugosicollis Ball. Im südl. Karste im ersten Frühjahre, s. h. Marolia variegata Bosco. Auf Nadelbäumen im Tarnowanerwalde, selten.

Anthicus tristis Schmidt. Am Meeresufer, nicht selten.

Anth. luteicornis Schm. In ausgetrockneten Sümpfen um Görz, n. s. Ochthenomus 1-fasciatus Bon. Am Wasser allenthalben, mitunter massenbaft.

Ochth. tenuicornis Rss. Auf Feldern, unter Steinhaufen, nicht häufig. Mordella 12-punctata Rss. Im Gebirge in morschem Holz und auf Blüthen, mitunter häufig.

Mord. 2-signata Rdtl. Um Görz auf Umbelliferen, gemein.

Emenadia flabellata $\mathrm{Fb}$. Um Görz $1 \delta^{\top}$ und 2 웅 auf Blüthen im Hochsommer.

Meloë autumnalis Ol. Im Spätherbst, besonders in Wäldern, n. s. Mylabris spartii Grm. Im südl. Karst, stellenweise in Masse.

Epicauta sibirica Pall. In Gemeinschaft der mitunter massenhaft auftretenden Ep. dubia, aber viel seltener. Exercierplatz bei Görz, Karst, hier bis 1400 Meter hoch.

Hapalus 2-maculatus L. Einige Ex. am Isonzo bei Görz.

Sitaris muralis Forst. $1 \mathrm{Ex}$. im Zimmer an der Wand, $1 \mathrm{Ex}$. auf einer Wiese gestreift.

Sit. apicalis Latr. 1 Ex. im Hochsommer auf Eryngium amethystinum. Xanthochroa carniolica Gistl. In der Stadt, namentlich wo Alleen sind, häufig. Fliegt erst nach 9 Uhr Nachts herum; bei Tage in Rindenspalten und unter Fenstergesimsen, hinter Hausthüren etc. versteckt, wo oft in Menge zu finden.

Oedemera subulata Ol. Im nördl. Karst gemein; durch sehr verdickte Schenkel von den deutschen Stücken unterschieden.

Oed. nobilis Scop. Um Görz allenthalben sehr gemein.

Oed. tristis Schm. Im nördl. Karst, besonders auf SambucusBlüthen in Masse.

Oed. anulata Grm. In Gemeinschaft der vorigen und womöglich noch häufiger.

Otiorhynchus pulverulentus Grm. Im Tarnowanerwald auf Nadelbäumen gemein.

ot. obsoletus Stierl. In den jul. Alpen, ziemlich häufig. 
Otiorhynchus inflatus Gyll. und pruinosus Grm. Um Görz allenthalben, nicht selten.

Ot. cardiniger Host. Allenthalben gemein, im Karst bis 1000 M. hoch. Ot. caudatus Rss. In den südl. Theilen des Coglio, z. B. bei Cormons, sehr häufig, um Görz selbst nur höchst vereinzelt.

Ot. obsitus Gyll. Im Gebirge weit verbreitet.

Ot. scabripennis Gyll. Im südl. Coglio bei Cormons nicht selten. Ot. istriensis Grm. Allenthalben gemein.

Ot. vochinensis Stierl. In den Vorbergen der jul. Alpen nicht selten. Ot. sulphurifer OI. Gemein, im Karst bis über 1200 Meter hoch. Ot. nobilis Grm. Im Hochgebirge auf Krummbolzkiefern stellenw. h. Ot. alutaceus Grm. Im ganzen Karst, aber immer nur einzeln. Ot. plumipes Grm. Im Gebirge, besonders gegen die Grenze Krains zu. Ot. crinipes Müll. Um Görz und im ganzen Karst gemein.

ot. cribrosus Grm. Im Tarnowanergebirge gemein.

Ot. scabrosus Mrh. Um Görz, besonders auf Epheu, nicht selten. Ot. frescati Boh. Auf Sumpfwiesen um Görz und Monfalcone.

Ot. foraminosus Boh. In den jul. Alpen etwa in 2000 M. Höhe z. h. Ot. elegantulus Grm. Im Tarnowanerwalde auf Buchengebüsch s. h. ot. crataegi Grm. Um Görz, aber vereinzelt.

Ot. signatipennis Gyll. Im Forste Panowitz bei Görz, besonders auf Juniperus und Crataegus sehr häufig. Mai.

Ot. illyricus Strl. Im Tarnowanergebirge ziemlich häufig.

Ot. chlorophanus Boh. Im Gebirge auf Cacalia alpina und Lamium orvala gemein.

Ot. corruptor Host. Gemein durch's ganze Gebiet.

Ot. fraxini Grm. Im nördl. Karst, aber nur an einzelnen Stellen. Ot. infernalis Grm. Im nördl. Karst, allenthalben nicht selten.

Ot. aterrimus Boh. In den jul. Alpen, nicht selten.

Ptochus 2-signalus Grm. Im ganzen Karst bis etwa 400 Meter hoch äufserst gemein.

Phyllobius cinereus Gyll. Im südl. Karst stellenweise häufig. Polydrusus sparsus Gyll. Um Görz im Frühjahr auf Crataegus gemein. Sciaphilus setulosus Grm. In den Vorbergen des nördl. Karstes, n. s. Barypeithes virguncula Seidl. Im Tarnowanerwalde auf Myosotis sylvatica häufig.

Caulostrophus Delarouzei Fairm. Im Hügellande um Görz, besonders auf eben ausschlagenden Eichen, nicht selten.

Sitones callosus Gyll. Um Görz in den Isonzo-Auen gemein, ebenso Brachycerus algirus $\mathrm{Fb}$,, in manchen Jahren häufig, seltener Br. lutosus Gyll. 
Hypera marmorata Cap. Im Tarnowanergebirge, selten.

Hyp. palumbaria Gm. Im Tarnowanerwalde auf Lamium orvala häufig. Cleonus morbillosus Fb. Im nördl. Karst, aber selten.

Lixus sanguineus Rss. Um Görz vereinzelt im ersten Frühjahr.

L. Ascanii L. und Myagri Ol. Häufig aus Rüben gezogen.

L. junci Boh. Im nördl. Karst in etwa 700 M. Höhe auf Artitschoken. Stolatus crinitus Boh. Auf blühender Pulicaria dysenterica stellenw. h.

Larinus cinerascens Gyll. Im nördl. Karst auf den Blüthen von

Centaurea rupestris gemein.

Anisorhynchus monachus Grm. Im südl. Karst, aber nicht häufig. Meleus Megerlei Pz. Im Tarnowanerwalde ziemlich häufig; ebenda Liosomus concinnus Boh. In Gesellschaft des Baryp.virguncula häufig. Lepyrus variegatus Schmdt. In den jul. Alpen, aber selten, ebenda Pissodes Gyllenhalli Gyll. Auf Krummholz häufig.

Bradybatus subfasciatus Grst. Auf blühendem Acer campestris um Görz häufig.

Cionus Olivieri Rnh. Im nördl. Karst auf Verbascum thapsus häufig. Nanophyes Chevrieri Boh. In der Ebene auf Lythrum salicaria gemein. Choerorhinus squalidus Fairm. Unter Obstbaumrinde um Görz, aber s. Apion holosericeum Gyll. In den niederen Theilen des Karstes, namentlich im südlichen, häufig.

Bruchus chinensis L. In der hiesigen Seidenbaustation mitunter in Masse.

$\mathrm{Br}$. debilis $\mathrm{Fb}$. Um Görz gemein.

$B r$. signaticornis Gyll. In Linsen ziemlich häufig.

Leptura cordigera Füsl. Um Görz selten, häufiger im südl. Karst, besonders auf Rubus - Blüthen.

Stenopterus ater L. Bei Cormons, selten.

Exilia timida Mén. $1 \mathrm{Ex}$. in Görz im Fluge gefangen.

Hesperophanes cinereus Vill. In Görz s. s., im südl. Coglio aber z. h. Callidium lividum Rss. Im ersten Frühjahr am Isonzo im Fluge.

Call. abdominale Bon. Am Isonzo auf blühendem Crataegus, selten. Clytus aegyptiacus $\mathrm{Fb}$. Um Görz, namentl. auf Achillea-Blüthen, häufig. Anaglyptus gibbosus F. Um Görz selten, im südl. Coglio zieml. häufig. Purpuricenus budensis Göze. Anfangs Juli im südl. Karst auf Blüthen von Clematis flammula.

Cerambyx velutinus Brull. Um Görz, sehr vereinzelt; miles Bon., nicht selten; nodulosus Grm., selten.

Exocentrus Clarae Muls. Auf Linden durch's ganze Gebiet, aber n. h. Belodera Genei Aug. Auf frisch ausgeschlagenen Eichen, besonders in den südlicheren Theilen der Provinz, nicht selten. 
Parmena baltea L. Auf Epheu, besonders im südl. Coglio, gemein. Dorcadion arenarium Scop. Im südl. Karst jedes zweite Jahr massenhaft.

Herophila tristis L. Allenthalben nicht selten. Nachtthier. Agapanthia cynarae Grm. Am Exercierplatz auf Disteln, nicht selten. Menesia 2-punctata Zbk. Auf Rhamnus alpinus, allenthalben häufig. Oberea melanura Grl. 3 Ex. auf Evonymus europaeus um Görz. Phytoecia cephalotes Küst. Im ganzen Karst eben nicht selten, aber wegen aufserordentlicher Flüchtigkeit sehr schwer zu fangen.

Phyt. rufipes Ol. Im Karst auf Ferulago galbanifera nicht selten. Donacia appendiculata Ahr. Auf Sparganium ramosum besonders im Herbst, sehr häufig.

Labidostomis lucida Grm. Auf jungen Buchentrieben um Görz, n. h. Lab. 3-dentata Lim. Um Görz, selten.

Cryptocephalus connexus Ol. Auf trockenen Wiesen im HochSommer, sehr häufig.

Chrysomela findelii Suffr. Im Karst allenthalben, aber nicht häufig. Chrys. cribrosa Ahr. Einige Ex. im Karst gefangen.

Malacosoma lusitanica L. Auf Compositen stellenw. äufserst häufig. Hyperaspis concolor Suffr. Auf trockenen Wiesen im Hochsommer gemein.

Die nachstehenden, beschriebenen Otiorhynchus sind ebenfalls von mir im Karst aufgefunden worden. 


\section{$2 \mathrm{BHL}$ Biodiversity Heritage Library}

Schreiber, Eg . 1885. "Sammelbericht aus dem Karst." Deutsche entomologische Zeitschrift 1885(2), 265-271. https://doi.org/10.1002/mmnd.48018850214.

View This Item Online: https://www.biodiversitylibrary.org/item/103411 DOI: https://doi.org/10.1002/mmnd.48018850214

Permalink: https://www.biodiversitylibrary.org/partpdf/235346

\section{Holding Institution}

Harvard University, Museum of Comparative Zoology, Ernst Mayr Library

\section{Sponsored by}

Harvard University, Museum of Comparative Zoology, Ernst Mayr Library

\section{Copyright \& Reuse}

Copyright Status: Public domain. The BHL considers that this work is no longer under copyright protection.

This document was created from content at the Biodiversity Heritage Library, the world's largest open access digital library for biodiversity literature and archives. Visit BHL at https://www.biodiversitylibrary.org. 